\title{
Evaluating Context Information Predictability for Autonomic Communication
}

\author{
Mirco Musolesi and Cecilia Mascolo \\ Department of Computer Science, University College London \\ Gower Street, London, WC1E 6BT, United Kingdom \\ \{m.musolesi|c.mascolo\}@cs.ucl.ac.uk
}

\begin{abstract}
Delay tolerant and mobile ad hoc networks, present considerable challenges to the development of protocols and systems. In particular, the challenge of being able to cope with their variability is an important one: sometimes the rate at which these systems change in terms of context (such as topology, colocation duration and availability and quality of the local resources) is very high and these changes are unpredictable. Knowledge of context could be used to improve the performance of such systems. For example, context information may be extremely useful to make routing decisions. Some recent approaches have successfully exploited context and prediction on future context condition to improve performance, for instance in terms of delivery ratio and delay.

In this paper, we present a model of predictability of context information and the design of a generic component implementing it. The component can be used to decide if (or in which measure) context is predictable. The model is based on the analysis of the time series representing the context information. In order to show how the component can be used in practice, we describe its integration in our Contextaware Adaptive Routing (CAR) protocol.
\end{abstract}

\section{Introduction}

Dealing with the variability and uncertainty is one of the major issues in many networked systems such as mobile ad hoc and delay tolerant networks [2]. The decentralization of the control and the movement of the hosts have a great impact on systems topology and, more generally, on their conditions. Recently, context information, such as colocation, local resource availability (i.e., memory and computing power), battery level, etc. have been used to improve the performance of such systems, for instance in terms of delivery ratio and delay.
There are situations, however, where context cannot be predicted. In these cases, using any prediction based techniques to improve performance of the system is completely ineffective. Complex and highly dynamic distributed systems would benefit from being able to assess when context prediction techniques can be successfully applied in order to improve the system behaviour. In this paper, we present a model of predictability of context information. We also describe a generic component implementing this predictability assessment model. The component can be used in very dynamic environments to introduce autonomy in deciding if (or how much) context is predictable. The technique adopted by the component is predicated on the analysis of the time series representing the context information. To our knowledge, this is the first attempt to offer a component for context predictability which could be used in dynamic distributed systems.

We describe the integration of such component in our Context-aware Adaptive Routing (CAR) protocol [8], a routing protocol for intermittently connected ad hoc networks, which chooses the best carrier for messages using context information (such as colocation with recipients, mobility rate and battery level). Our predictability component allows each node to dynamically decide when the prediction approach at the basis of CAR is useful and when not. In this way each node can autonomically decide on when to use CAR or when, instead, use another protocol, such as an epidemic-like approach [11].

This paper is structured as follows: in Section 2 we present our model for the assessment of the predictability of context information and the design of a software component implementing it. In Section 3, we discuss the integration of the predictability component in the CAR protocol as case study, also showing simulation results using different mobility models. Section 4 is focussed on the comparison of the proposed model with the state of the art and its possible impact on existing systems. Finally, Section 5 concludes the paper. 


\section{Evaluating Predictability of Context}

We refer to context as the set of quantitative indicators representing a networked system that are useful to achieve a certain goal. A typical example is the delivery of messages in mobile ad hoc networks. In these settings, possible examples of context information are the patterns of colocation of hosts, the movements of mobile nodes (that could be expressed in terms of change degree of connectivity) and the residual battery power of devices.

We assume that a certain type of context information, such as the change degree of connectivity of a certain host, at time $t$ can be described using values in a given range (e.g., $[0 \ldots 1]$ ). For example, colocation can be represented using 1 to indicate that a certain host is in the same transmission range, 0 otherwise; battery level can be normalized by dividing the current energy level by the battery capacity in order to have a value in the range [0..1]. Given these definitions, the temporal evolution of each context attribute can be represented using a time series of the values that it assumes in subsequent instants of time.

\subsection{Predictability Monitoring Component}

We have designed a component that receives in input the observed value (at time $t$ ) and the corresponding predicted value (calculated at time $t-1$ ) of a particular context indicator (such as the current battery level) and is able to decide if the prediction model (in the case of our prototype system, a Kalman filter based predictor [3]) is able to forecast the next value of the time series with a given accuracy that can be set by the developer.

To estimate the predictability of context, we exploit a technique based on the analysis of the time series of the prediction errors [1]. More specifically, we are interested in the analysis of the so-called residuals, defined as the differences between the observed values at time $t$ and the onestep-ahead forecast for this same value at time $t$. More formally, given the observed value $y(t)$ and the predicted value $\widehat{y}(t)$ we define the residual at time $t$ as follows:

$$
z(t)=\widehat{y}(t)-y(t)
$$

Since we are considering time series, the residuals are ordered in time and, can therefore be treated as a time series too. In particular, our analysis is based on the correlogram (also called sample autocorrelation function) of residuals. A correlogram is a diagram in which the sample autocorrelation coefficients $r_{k}$ are plotted against the lag $k$ for $k=0,1 \ldots, M$, where $M$ is usually much less than the number of samples $N$ taken into consideration.

The autocorrelation coefficient of $z(t)$ at lag $k, r_{k}$, is defined as follows:

$$
r_{k}=\frac{\sum_{t=1}^{N-k}(z(t)-\mu)(z(t+k)-\mu)}{\sum_{t=1}^{N}(z(t)-\mu)^{2}}
$$

with $\mu=\sum_{t=1}^{N} \frac{y(t)}{N}$

Values of $r_{k}$ close to zero indicate that the values of the time series $z(t)$ are scarcely correlated at a distance $k$. When the prediction model provides a good estimation of the future trend of the context information, the standardised forecast errors are serially uncorrelated. Therefore, the correlogram of forecast errors should reveal insignificant serial correlation. In other words, if we have a good prediction model, then we expect the residuals to be random (i.e., the time series $\{z(t)\}$ forms a random process) and close to zero. If the residuals $\{z(t)\}$ forms a purely random process, their correlogram is such that each autocorrelation coefficient is approximately normally distributed, with mean 0 and variance $\frac{1}{N}$ for sufficiently large values of $N$.

Let us then suppose that $z_{1}, z_{2}, \ldots, z_{N}$ are observations on independent and identically distributed random variables with arbitrary mean. It can be shown that $r_{k}$ is asymptotically normally distributed and that

$$
\begin{aligned}
& E\left(r_{k}\right) \approx-\frac{1}{N} \\
& \operatorname{Var}\left(r_{k}\right) \approx \frac{1}{N}
\end{aligned}
$$

We can use this result to check the randomness of the time series of the residuals. Let us suppose that we are interested in $95 \%$ confidence interval in our calculation. In this case the range of the confidence interval is

$$
\left[-\frac{1}{N}-\frac{2}{\sqrt{N}}, \frac{1}{N}+\frac{2}{\sqrt{N}}\right]
$$

This interval can be further approximated as

$$
\left[-\frac{2}{\sqrt{N}}, \frac{2}{\sqrt{N}}\right]
$$

Observed values of $r_{k}$ which fall outside these limits are significantly different from 0 at the $5 \%$ level. Therefore, considering a degree of confidence of $95 \%$, no more than $5 \%$ of the values should be outside the range $\left[-\frac{2}{\sqrt{N}}, \frac{2}{\sqrt{N}}\right]$. For example, if the first 20 values of $r_{k}$ (i.e., $r_{1}, r_{2}, \ldots, r_{k}$ ) are taken into consideration, one value outside the range may be expected, even if the data are really random. If we obtain more than one of the values of $r_{k}$ outside the range, then we can deduce that the time series of the residuals is not random. Therefore, the prediction model that we are using is not sufficient to forecast the future behaviour of the 
time series representing the context information taken into consideration.

At the same time, the values of $z(t)$ should be close to zero. In order to test this, we consider the normalised value of $z(t)$ by dividing it by 100 . We then define an acceptable percentage error $\zeta$ (for example equal to $0.1 \%$ ). The value of $\zeta$ can be chosen according to the developer's application requirements. If the $z(t)>\zeta$, once again we derive that the prediction model does not provide good forecast results.

To summarize, the prediction component will return true if the $z(t)>\zeta$ and the time series of the last $M$ values of the residuals is random. If there are not enough samples, the component is not able to derive any information about the predictability of the time series and, therefore, it will notify this fact to the system.

\section{Case Study: Integration of the Predictor Component in the CAR Protocol}

In order to show how the predictability component can be integrate into dynamic distributed systems, we show how we integrate it into our Kalman Filter [3] based Contextaware Adaptive Routing protocol (CAR) [8]. CAR allows messages to be delivered also in environments where disconnections or host partitions are often present; the protocol relies on the selection of the hosts are able to act as best message carriers in order to enable transitive communication. As it can be seen from the performance results illustrated in [8], this protocol is very effective when the context is somehow predictable. In case context is not predictable, the protocol could only be detrimental to the system, as it will be shown in this paper. It is therefore of paramount importance to be able to distinguish these two situations and to be able to allow autonomic adaptation of the hosts in terms of which routing protocol to use based on the context (and its predictability).

\subsection{The CAR protocol at a Glance}

We now present the main characteristics of the CAR protocol, focussing on the aspects related to the prediction of the evolution of the context information. CAR is a communication protocol for intermittently connected networks. It is based on the idea of transitive communication using intermediate nodes by means of store-and-forwarding mechanisms. If the recipient of the message is in the same connected network of the sender, the message is delivered using the underlying synchronous protocol such as DSDV [10]. If the recipient is not in the same connected network, the message is delivered by means of a carrier. Therefore, the general problem from the point of view of the sender of a message is to find the host with the best delivery probability (i.e., the best carrier). In CAR, this choice relies on the application of forecasting techniques for the analysis of the evolution of the context described using a set of context attributes (battery, mobility, colocation).

The process of prediction and evaluation of the context information can be summarized as follows. Each host calculates its delivery probability. This process is based on the prediction of the future values of the attributes describing the context and on the composition of these estimated values using multi-attribute utility theory [4]. The calculated delivery probabilities are periodically sent to the other hosts in a connected network (with a horizon of some hops), as part of the update of routing information. Each host maintains a logical forwarding table of tuples describing the next logical hop (best carrier), and its associated delivery probability, for all known destinations.

In the original version of CAR described in [8], we did not consider a key aspect of the problem, the predictability of the context information. In fact, the Kalman filter based used in CAR is not able to provide accurate forecasts in any mobile scenarios, such as in presence of time series characterised by a high degree of randomness. A quantitative measure of the quality of the prediction is then essential to cope with the cases when accurate predictions are not possible. In fact, in these cases, the CAR protocol is not the best choice for the routing. Using the predictability component, each host is then able to decide whether to choose a different protocol when prediction is not possible, such as an epidemic protocol [11].

\subsection{Simulation of the System}

We evaluated the performance of the prediction component integrated in CAR using the OMNeT++ [12] simulation framework. In particular, we studied the predictability of the colocation and the change degree of connectivity using the Random Way Point (RWP) and the Community based mobility models [9]. As discussed in [9], the variability of the colocation and mobility of the hosts in scenarios generated by the Community based mobility model is not random but based on deterministic values given in input and follow precise patterns.

We consider a mobile scenario composed of 32 hosts in $1 \mathrm{~km} \times 1 \mathrm{~km}$ area. We assume a free space propagation model and the use of an omnidirectional antenna. The transmission range is equal to $200 \mathrm{~m}$. We considered 100 messages with a simulation time equal to 600 seconds. The messages were sent after 40 seconds, in order to allow for the settling of initial routing table exchanges, and the intervals between each message were modeled as a Poisson process, with $\lambda=5 s^{-1}$. The sender and receiver of each message are chosen randomly. Each message has a field that is similar to a time to live value that is decreased each time that the message is transferred to another host (the initial 
value being 15). Moreover, in this case, we also introduced a split horizon mechanism to prevent messages from being retransmitted unnecessarily. The buffer for each node was set to 100 messages (i.e., infinite buffers). We considered a $5 \%$ error confidence interval and and a maximum prediction error equal to $0.1 \%$. The number of samples used in the calculation of the randomness of the time series of the residuals is 60 with a maximum lag equal to 20 . We used multiple runs to achieve a statistically valid number of simulations.

\subsubsection{Simulation Results}

Figure 1 and 2 show the performance of the CAR protocol respectively in terms of delivery ratio and delay using the RWP and the Community based mobility models. As far as the delivery ratio is concerned, the performance in mobile scenarios generated using the Community based model are considerably better than in those generated using the RWP model. This can be explained by considering that the predictability of the time series of the change rate of connectivity and of the colocation between the hosts degrades as the speed increases (Figure 3 and 4). More specifically, in Figure 3, we plotted the average percentage of hosts (normalized in the range $[0,1]$ ) for which valid prediction were available. In Figure 4, instead, we plotted the average percentage of time during which valid predictions of the change rate of connectivity were available.

With respect to the delivery delay, in the scenarios generated using the Community based model, the performance of CAR improves as the speed increases and then decreases again for values of speed greater than $10 \mathrm{~m} / \mathrm{s}$. This is due to the fact that the predictability of the context information also decreases for these values of speed and after a certain threshold, the Kalman filter used in CAR is not able anymore to cope with the variability of the system.

Instead, in scenarios generated using the RWP model, the performance degrades steadily as the speed increases. Since CAR relies on DSDV for delivering messages in the same connected portion of the network of the sender, the performance degrades as the speed increases (due to the inherent characteristics of DSDV) and, at the same time, there is no improvement due to the asynchronous dispatching operated by the message carriers. In fact, as it is possible to see in Figure 3 and 4, both the predictability of the time series of the colocation and the change degree of connectivity degrade considerably. The better performance in the case of the speed equal to $2 \mathrm{~m} / \mathrm{s}$ and $4 \mathrm{~m} / \mathrm{s}$ can be explained by considering the fact that the Community based mobility model generates network topologies characterized by the presence of disconnected networks of hosts, whereas the RWP ensures a better connectivity.

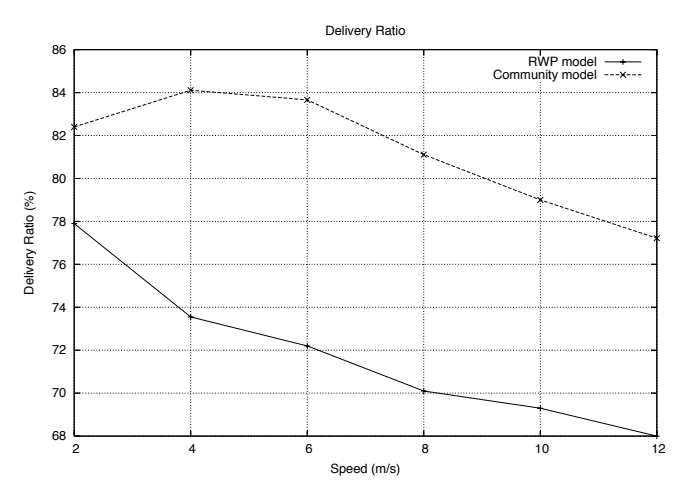

Figure 1. Delivery ratio vs Speed

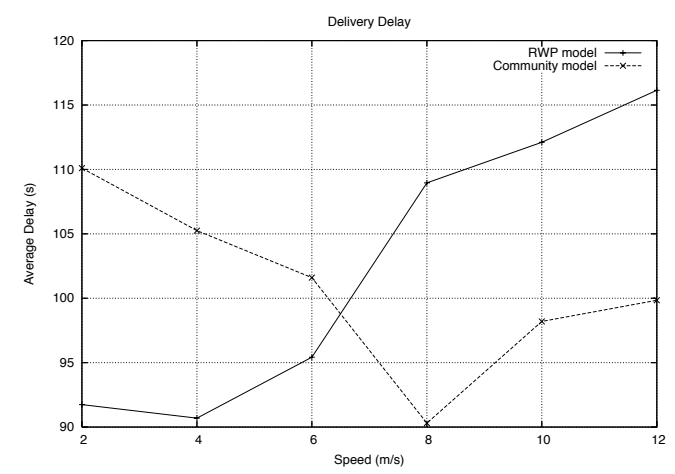

Figure 2. Average delivery delay vs Speed

\section{Related Work and Discussion}

In the recent years, prediction techniques have been applied in different areas of computer science and in particular in the field of dynamic distributed systems and mobile networking.

Systems for delay tolerant communication based on the prediction of movements or other resource indicators include [7, 13]. However, these works do not rely on the analysis of time series like CAR and do not take into consideration the problem of the predictability of the indicators used to improve the system performance.

In [6] the authors analyze the application of various types of filters to forecast network performance; however, they do not take into consideration the problem of evaluating the actual predictability of the observed systems. More recently, in [5], Kalman filter techniques have been used to extract the movement of the users. Also in this work, the aspects related to the predictability of the users' mobility are not 


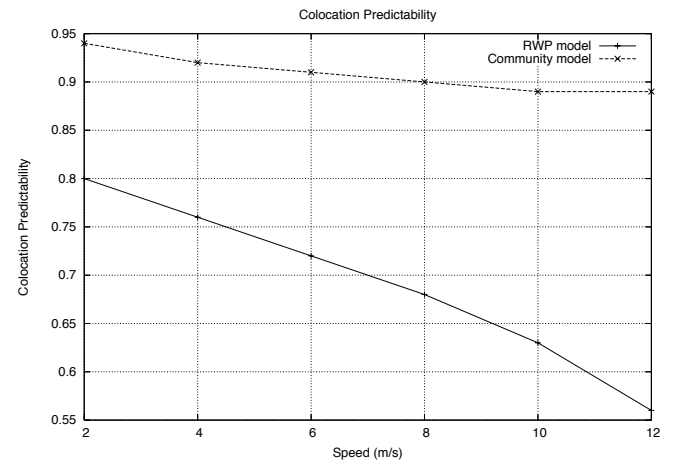

Figure 3. Colocation predictability vs Speed

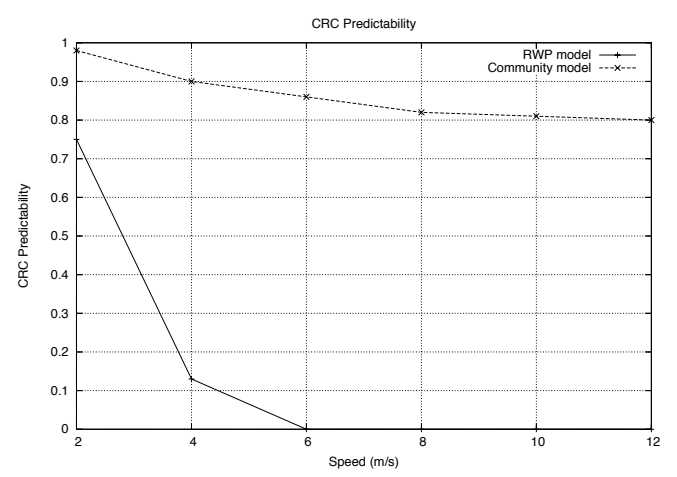

Figure 4. Change degree of connectivity predictability vs Speed

directly considered.

We believe that these systems can benefit from the integration of our component in order to make alternative decisions when prediction is not possible. Our approach is lightweight, since it requires to store only a small number of the past values of the context information. Moreover, it is easy to integrate with virtually all possible prediction techniques based on the analysis of time series. Furthermore, we believe that it may be applied in many other fields in computer science where similar prediction techniques are (or can be) used such as peer-to-peer systems and grid computing.

\section{Concluding Remarks}

We have presented our component for predictability assessment of context information. We have first indicated why such a component can be useful, then we have described the details of the model. In order to illustrate the integration of the component in practice, we have shown how it was used to dynamically assess the prediction of a context-aware routing protocol which uses context forecast to determine the best message carrier. We have evaluated the approach using a mobility model validated using data from real traces of movement and show that the component effectively realizes when the movement data are random and a prediction would not work.

\section{References}

[1] C. Chatfield. The Analysis of Time Series An Introduction. Chapman and Hall, 2004.

[2] K. Fall. A delay-tolerant network architecture for challenged internets. In Proceedings of SIGCOMM'03, August 2004.

[3] R. E. Kalman. A new approach to linear filtering and prediction problems. Transactions of the ASME Journal of Basic Engineering, March 1960.

[4] R. L. Keeney and H. Raiffa. Decisions with Multiple Objectives: Preference and Value Tradeoffs. Wiley, 1976.

[5] M. Kim, D. Kotz, and S. Kim. Extracting a mobility model from real user traces. In Proceedings of INFOCOM'06, Barcelona, Spain, April 2006.

[6] M. Kim and B. Noble. Mobile network estimation. In Proceedings of MobiCom '01, pages 298-309, New York, NY, USA, 2001. ACM Press.

[7] A. Lindgren, A. Doria, and O. Schelén. Probabilistic routing in intermittently connected networks. In Proceedings of SAPIR 2004, August 2004.

[8] M. Musolesi, S. Hailes, and C. Mascolo. Adaptive Routing for Intermittently Connected Mobile Ad Hoc Networks. In Proceedings of the 6th International Symposium on a World of Wireless, Mobile, and Multimedia Networks (WoWMoM 2005). Taormina, Italy. IEEE press, June 2005.

[9] M. Musolesi and C. Mascolo. A community based mobility model for ad hoc network research. In Proceedings of the 2nd ACM/SIGMOBILE International Workshop on Multi-hop Ad Hoc Networks: from theory to reality (REALMAN'06). ACM Press, 2006. To appear.

[10] C. E. Perkins and P. Bhagwat. Highly Dynamic DestinationSequenced Distance-Vector Routing (DSDV) for Mobile Computers. In Proceedings of SIGCOMM'94, pages 234-244, August 1994.

[11] A. Vahdat and D. Becker. Epidemic Routing for Partially Connected Ad Hoc Networks. Technical Report CS-2000-06, Department of Computer Science, Duke University, 2000.

[12] A. Varga. The OMNeT++ discrete event simulation system. In Proceedings of ESM'2001, Prague, 2001.

[13] Y. Wang and H. Wu. DFT-MSN: The Delay/Fault-Tolerant Mobile Sensor Network for Pervasive Information Gathering. In Proceedings of INFOCOM'06, Barcelona, Spain, April 2006. 